lesions on bone scan. 4 patients with metastatic bony lesions did not have a bone marrow performed, while the other 3 did not have evidence of disease in the bone marrow aspirate or trephine.

Conclusions Although our numbers are small, there is a high correlation between bony metastases identified by Technetium 99 bone scan and bone marrow aspirate and trephine positivity. Further prospective evaluation is required to determine whether a bone marrow examination adds any value to the initial staging of Ewings sarcoma over currently available imaging techniques and whether it can be omitted from the list of initial staging assessments.

\section{G404 MODELS OF KEY WORKING IN CHILDREN'S CANCER CARE: PROFESSIONALS AND PARENTS VIEWS}

${ }^{1} \mathrm{~A}$ Martins, ${ }^{1} \mathrm{~S}$ Aldiss, ${ }^{1,2} \mathrm{~F}$ Gibson. ${ }^{1}$ Department of Children's Nursing, London South Bank University, London, UK; ${ }^{2}$ Great Ormond Street Hospital for Children NHS Foundation Trust (GOSH), London, UK

\subsection{6/archdischild-2015-308599.358}

Purpose The importance of the key worker role in promoting continuity and coordination of care for children with cancer and their families is recognised but evaluation of such services is less well developed with little information available about different patterns of provision and the determinants for success. Therefore, our aims were: identify models of key working and influencing factors; analysis of professionals and parents' views of the impact of the key worker role on families' experiences.

Methods Thirty-six semi-structured individual interviews and one focus group $(\mathrm{n}=12)$ with key workers and twenty-eight interviews with parents were conducted. Multiple case study method was used to identify the models of key working developed and the impact on families' experiences.

Results Four models of care were identified. These models are organised along two dimensions - presence/absence of home visits and presence/absence of direct delivery of clinical care. Key worker involvement in care coordination and home visits was influenced by the resources available, in the hospital and in the community, these included staff and caseload numbers. Parents viewed the key worker role as a reliable, consistent, approachable source of support. The support was tailored to parent's needs and included information and advice, emotional and practical support. Having a main point of contact was highly valued by parents. In particular, parents identified the proactive and responsive nature of the service as central to the value they placed on it. However, some parents recognised the pressures on the key worker to support all the families in their caseload. Consequently, some parents described rationing their demands on the service in the context of other people with greater needs or feeling sometimes that the key worker was not available.

Conclusion The key worker role is instrumental in enabling families and patients to access and navigate services. The complexity of families' needs throughout the cancer journey is reflected in the complexity of the key worker role. Regardless of the model of care developed, core characteristics of the role and its impact on families' experiences were identified, these include: coordination of care, being a main point of contact and information.

\section{G405 \\ DEFINING HEALTHCARE PROFESSIONAL COMPETENCE FOR WORKING WITH TEENAGERS AND YOUNG ADULTS WITH CANCER - A BRIGHTLIGHT STUDY}

${ }^{1,2}{ }^{2} \mathrm{RM}$ Taylor, ${ }^{1} \mathrm{~N}$ Aslam, ${ }^{3} \mathrm{R}$ Feltbower, ${ }^{4} \mathrm{R}$ Raine, ${ }^{5} \mathrm{JS}$ Whelan, ${ }^{6} \mathrm{~F}$ Gibson. ${ }^{1}$ Cancer Clinical Trials Unit, University College London Hospitals NHS Foundation Trust, London, UK; ${ }^{2}$ School of Health \& Social Care, London South Bank University, London, UK; ${ }^{3}$ Division of Epidemiology \& Biostatistics, School of Medicine, University of Leeds, Leeds, UK; ${ }^{4}$ Department of Applied Health Research, University College London, London, UK; ${ }^{5}$ Department of Oncology, University College London Hospitals NHS Foundation Trust, London, UK; ${ }^{6}$ Great Ormond Street Hospital for Children NHS Foundation Trust, London, UK

\subsection{6/archdischild-2015-308599.359}

Aims Teenage and young adult (TYA) medicine is emerging as a distinct speciality, acknowledging the core tasks required to enable a young person to transition successfully to adulthood. As healthcare education is subdivided into adult or child focusedtraining, a framework is needed to reflect the specific competencies required to care for this population. The aim was to provide international consensus on the competencies required by healthcare professionals to provide specialist cancer care for TYA.

Methods A modified, international e-Delphi survey was conducted over 2 rounds. Experts were defined as professionals having worked in TYA cancer care for more than 12 months. They were identified through publications and invitations via professional organisations. The content of round 1 was informed by UK pilot work and a workshop undertaken by the professional organisation, TYAC. There were 87 closed-ended questions with responses on a 9-point Likert scale and further open-ended responses to identify other skills, knowledge and attitudes. Round 2 contained only items with no consensus in round 1 and suggestions of additional items of competency. Consensus was defined as a median score ranging from 7-9.

Results A total of 179 registered to be members of the expert panel, of which valid responses were available for $158(88 \%)$ in round 1 and $136(86 \%)$ for round 2 . The majority of participants were nurses $(35 \%)$ or doctors $(39 \%)$ from Europe $(55 \%)$ or North America (35\%). All 87 items in round 1 reached consensus with an additional 15 items identified for round 2 . The most important skill was reported as "being able to identify the impact of disease on young people's life"; the most important area of knowledge was "know about side-effects of treatment and how this might be different to those experienced by children or older adults"; "ability to talk about difficult issues" was the most important communication skill and honesty the highest rated attitude.

Conclusions The process has been successful in identifying a high degree of consensus for competencies and skills to be used of training for professionals working with TYA.

\section{G406 COPING WITH CANCER - SUPPORTING YOUNG PEOPLE'S RESILIENCE}

HL Gravestock, S Malik. CLIC Sargent, London, UK

\subsection{6/archdischild-2015-308599.360}

Aims

- To better understand the help-seeking behaviour and information needs of young people with cancer, and how quality support and information can help them build and maintain 